The Omics Dashboard for Metabolomics Data

Peter D. Karp, Suzanne Paley, Paul O'Maille

SRI International

ecocyc.org

biocyc.org

metacyc.org

\title{
(1). BIOCYC
}

Database Collection 


\section{Overview of Omics Dashboard}

- New interactive tool for analysis of omics data

- Metabolomics

- Gene expression

- Proteomics

- Use cases:

- Quickly survey how all cellular systems are responding to a stimulus

- Examine specific pathways, subsystems of interest

- Gauge relative metabolite levels

- Available at BioCyc.org, and with downloadable Pathway Tools software

- Paley et al., Nucleic Acids Research 2017 


\section{BioCyc.org Overview}

- 11,000 Pathway/Genome Databases for sequenced organisms

- Predicted metabolic reactions, pathways, metabolomes

- Atom mappings, metabolic models

- Curated from 66,000 publications

- Extensive bioinformatics tools for metabolomics analysis

- Paint metabolomics data onto individual pathways, multi-pathway diagrams, full metabolic maps

- SmartTables to manipulate metabolite sets (e.g., map to pathways)

- Karp et al, Metabolites 5:291 2015
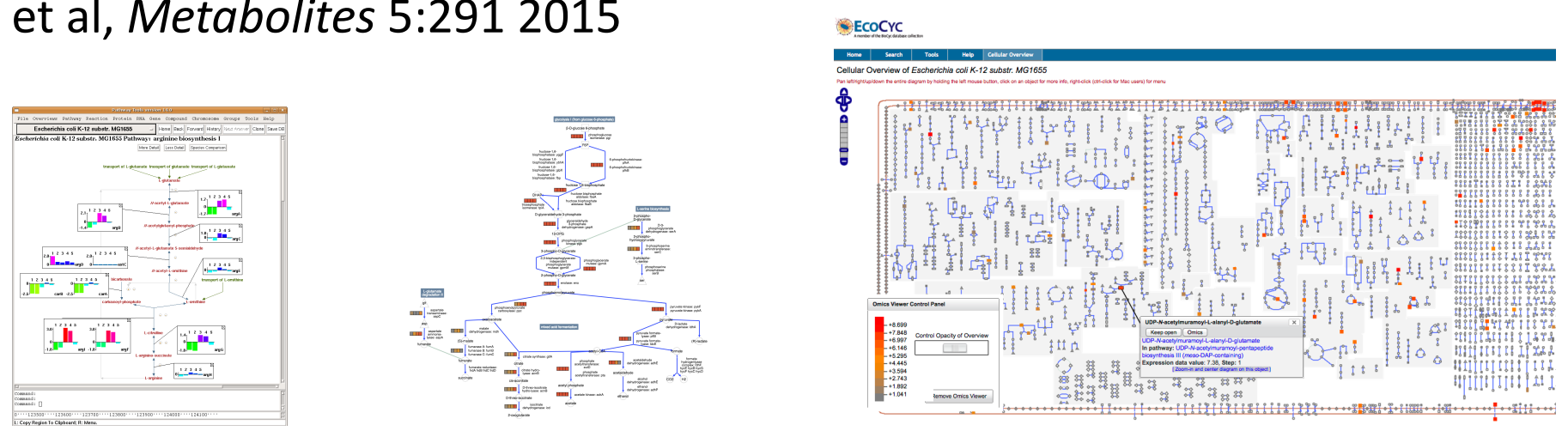


\section{Organization of Omics Dashboard}

- Panels summarize omics data for multiple cellular systems

- Each panel contains a set of plots (subsystems)

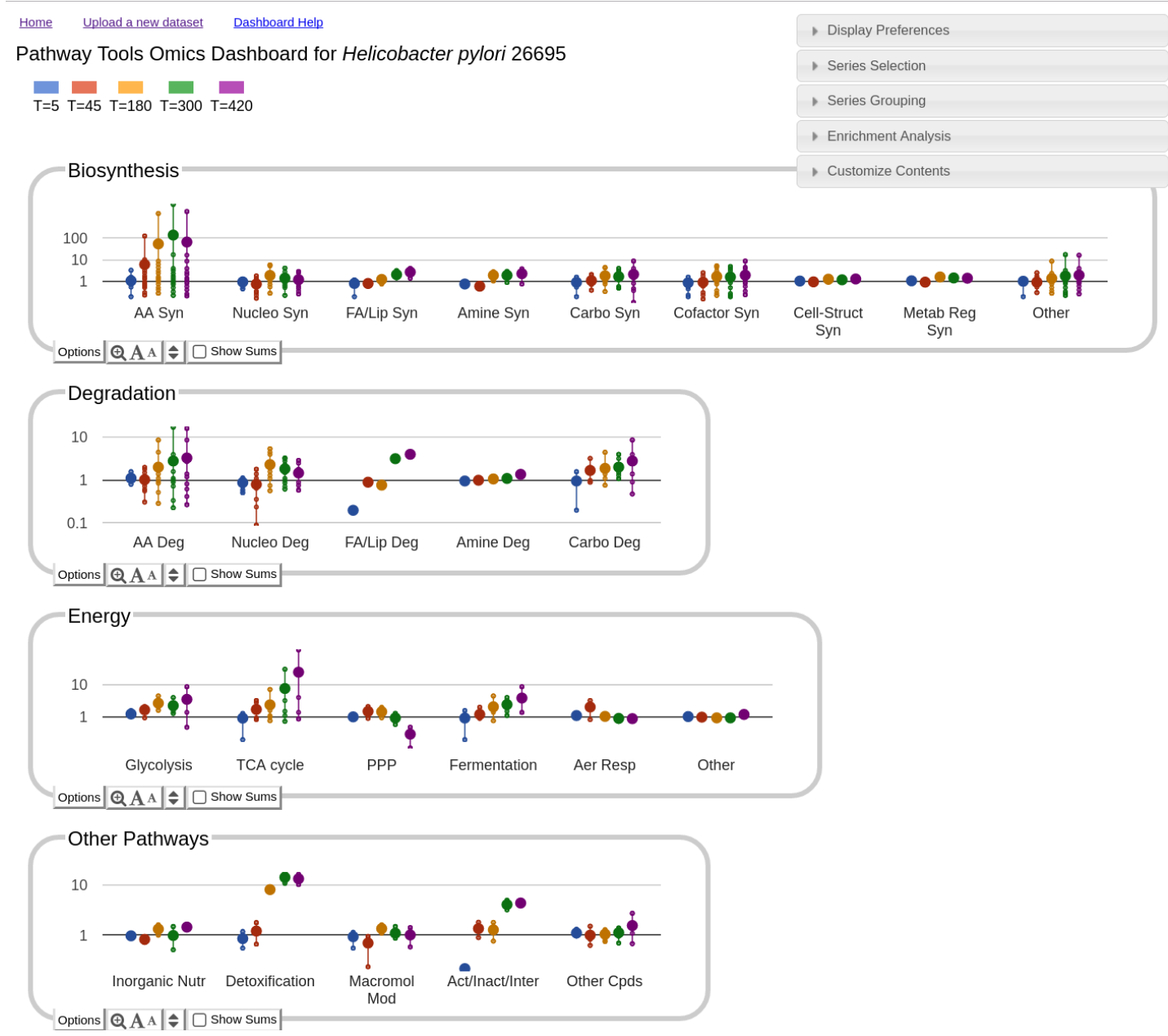




\section{Omics Dashboard Panels}

- Panels and plots available configured automatically for current organism and current dataset

- Plots defined from MetaCyc pathways and pathway classes, Gene Ontology terms

- User can add or remove plots from any panel

- Many non-metabolic panels for gene-expression data

- Click on a plot to drill down for more information 


\section{Using the Dashboard}

- Apply normalization and significance calculations before uploading data, if applicable

- Import data as a column-delimited file

- Metabolite names/IDs in first column

- Specify which other columns contain data of interest

- Compare all metabolites with only significantly changed metabolites

- Types of data values

- Fold change values

- Absolute quantities (counts, areas, intensities)

- P-values from replicate analysis (used for enrichment analysis) 


\section{Invoking the Omics Dashboard}

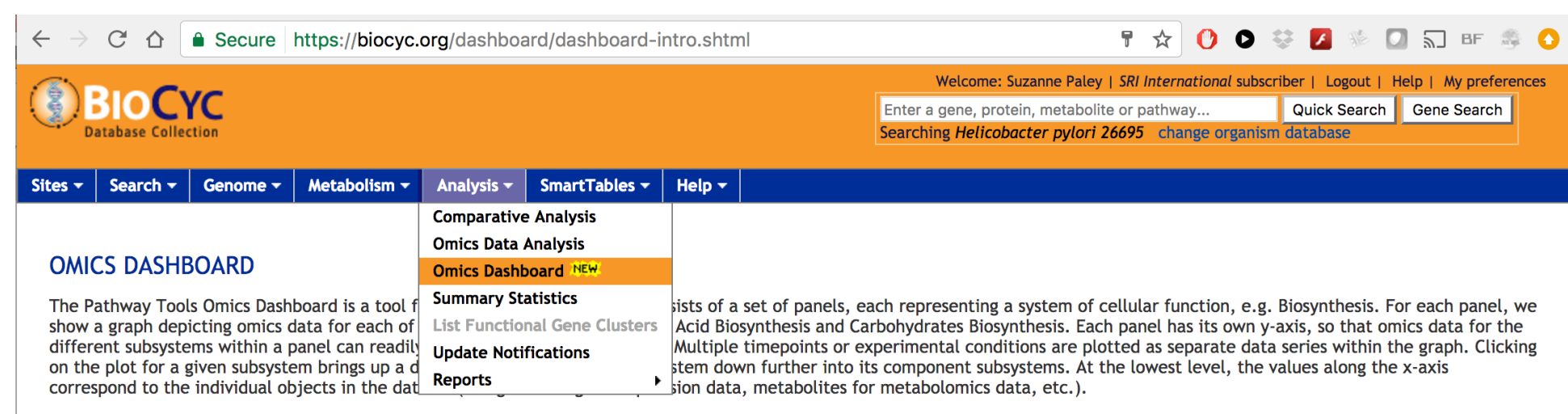

See the Omics Dashboard Help document for more information, or see an example of the Omics Dashboard in action using a sample dataset.

Choose one of the following options to specify omics data for the Omics Dashboard:

Your most recently uploaded omics dataset

- Upload a tab-delimited file

Select a file containing experimental data: Choose File Mtz_p-values ...Id change.txt
Items in the first column of the file are: Compound names and/or identifiers

Items in the first column of the file are: Compound names and/or identifiers
Data column(s) to use: (The first column is numbered 0) 2-11

Select type of values: Relative 0 ?

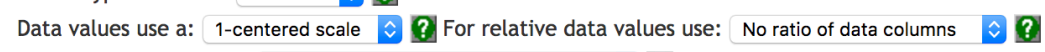

Choose a color scheme: Orange to blue with a maximum cutoff $\hat{v}$ ?

Maximum cutoff is: 5

Note that the color scheme selected here will not be used for the dashboard graphs, but only for inclusion of omics data in pathway diagrams that are generated from the dashboard.

Submit

Import data from a SmartTable

Note: While uploading a large dataset to the dashboard may take some time to process initially, if you are experiencing ongoing performance issues while interacting with the dashboard, we recommend you try one of the following:

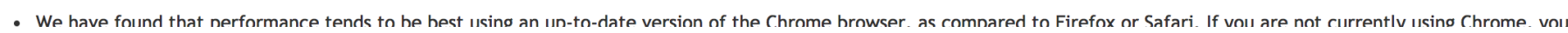




\section{Helicobacter pylori Dataset - MoA of antibiotics}

- Unpublished data courtesy of Paul O’Maille (SRI Biosciences)

- Time series metabolomics investigation of metronidazole action against $H$. pylori (compared to DMSO control)

- Current MoA:

- Metronidazole (prodrug) converted to radicals in anaerobic pathogens

- Metronidazole radicals induce DNA breakage through random attack ${ }^{1}$

- Metabolomics and Dashboard - new tools, new insights 


\section{Helicobacter pylori Dataset - MoA of antibiotics}

- Study design:

- H. pylori cultures exposed to metronidazole (or DMSO control)

-5 biological replicates taken at $T=5,45,180,300,420$ minutes

- Metabolon data collection and processing:

- Global (non-targeted) metabolite analysis of samples via LC/GC-MS platform

- Replicates averaged, and fold changes computed relative DMSO control

- Significance p-values computed using Two Way ANOVA with Contrasts 


\section{Helicobacter pylori Dataset - Metabolome Coverage}

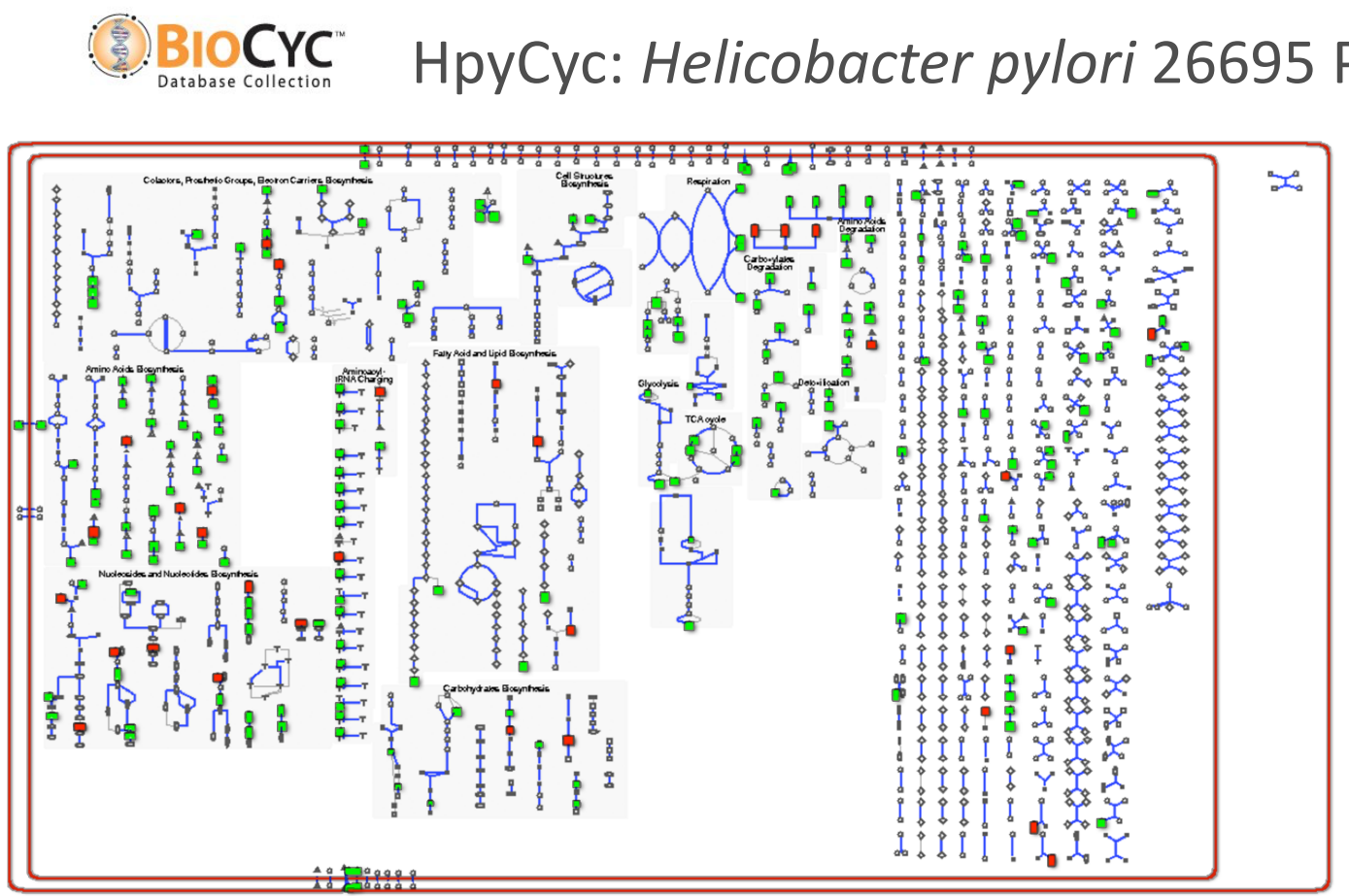

- 318 H. pylori

metabolites identified

- 103 matches out of 602 total HpyCyc metabolites

- $17 \%$ coverage of $H$. pylori metabolome

Cellular overview - colored nodes are experimentally measured metabolites where statistically significant (red) and nonsignificant (green) changes are indicated. 


\section{Metabolite Enrichment Analysis}

- Which pathways have more perturbed metabolites than expected by chance?

- User specifies

- A p-value column associated with each timepoint

- Threshold (0.05 in this example)

- Multiple hypothesis correction function

- Compute enrichment score for every pathway

- Fisher-exact hypergeometric test

- Enrichment score for a pathway $=-\log _{10}(p$-value $)$

- Panels show

- Enrichment scores for each pathway

- Highest component subsystem score 


\section{Standard Display vs. Enrichment Mode Display}

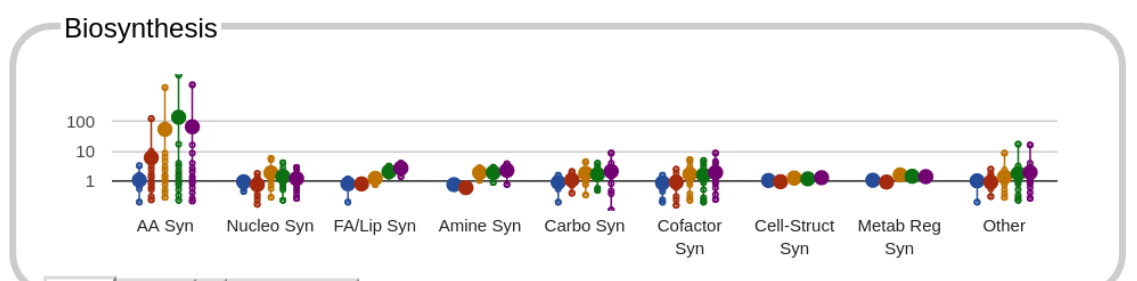

options $|\underline{Q A A}| \hat{\mid} \mid \square$ show Sums
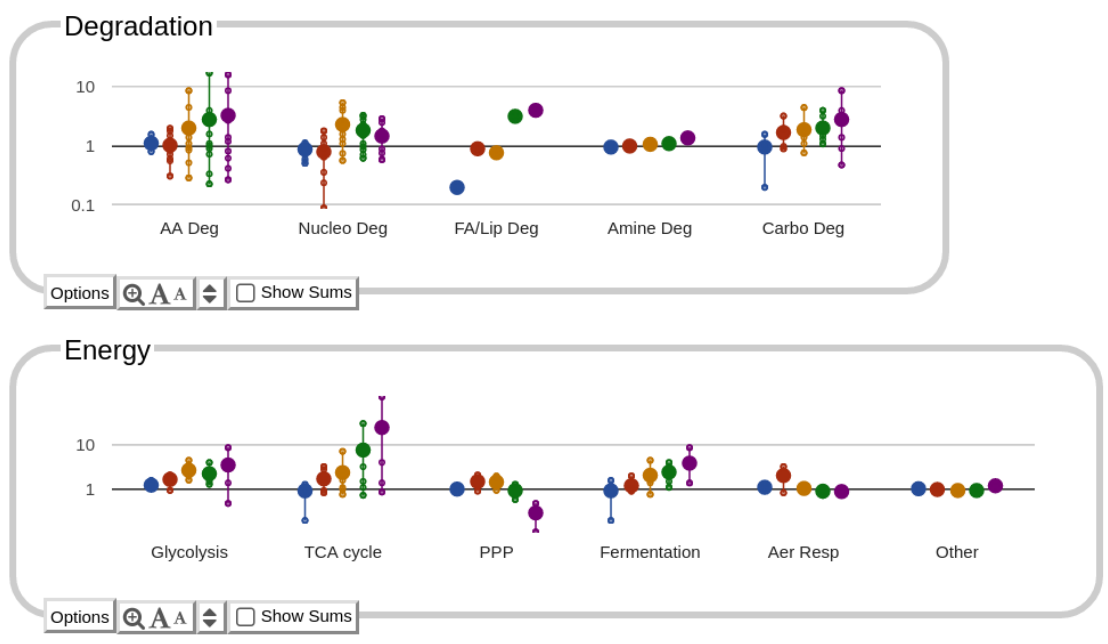

options $\mid$ QAA $|\hat{\mid}| \square$ Show Sums

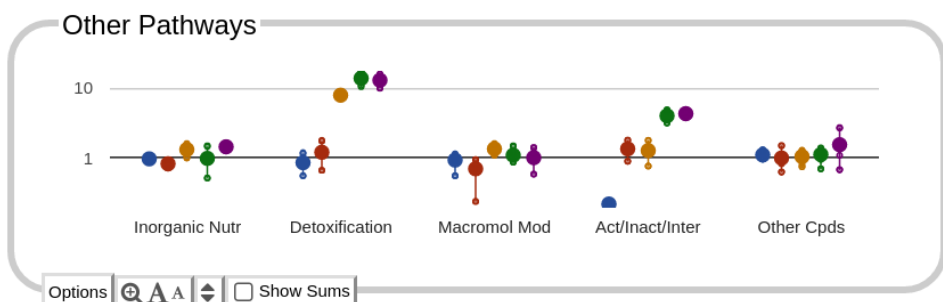

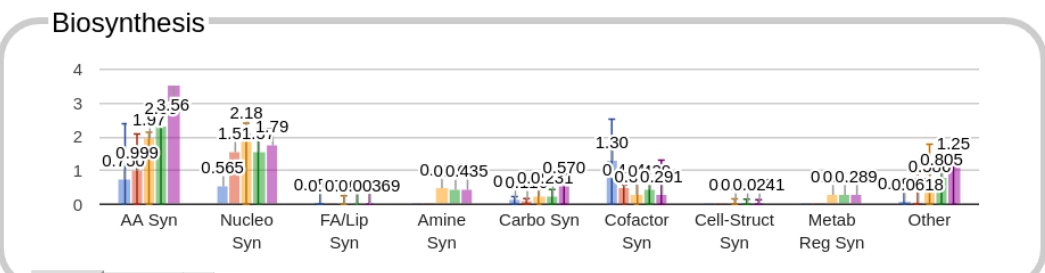

options $\mathbf{Q A A} \mid \hat{A}$
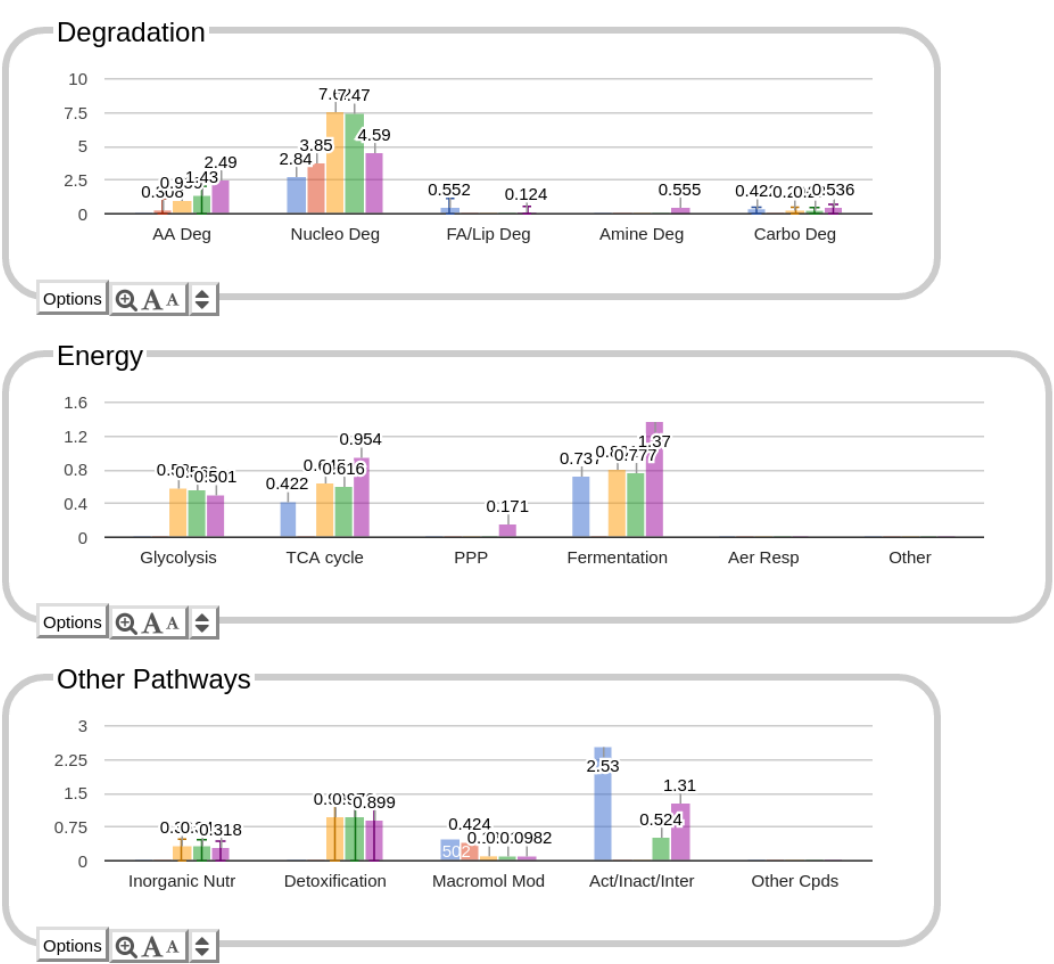


\section{Sulfur-Containing Amino Acids Disrupted During Early Phase}

Action

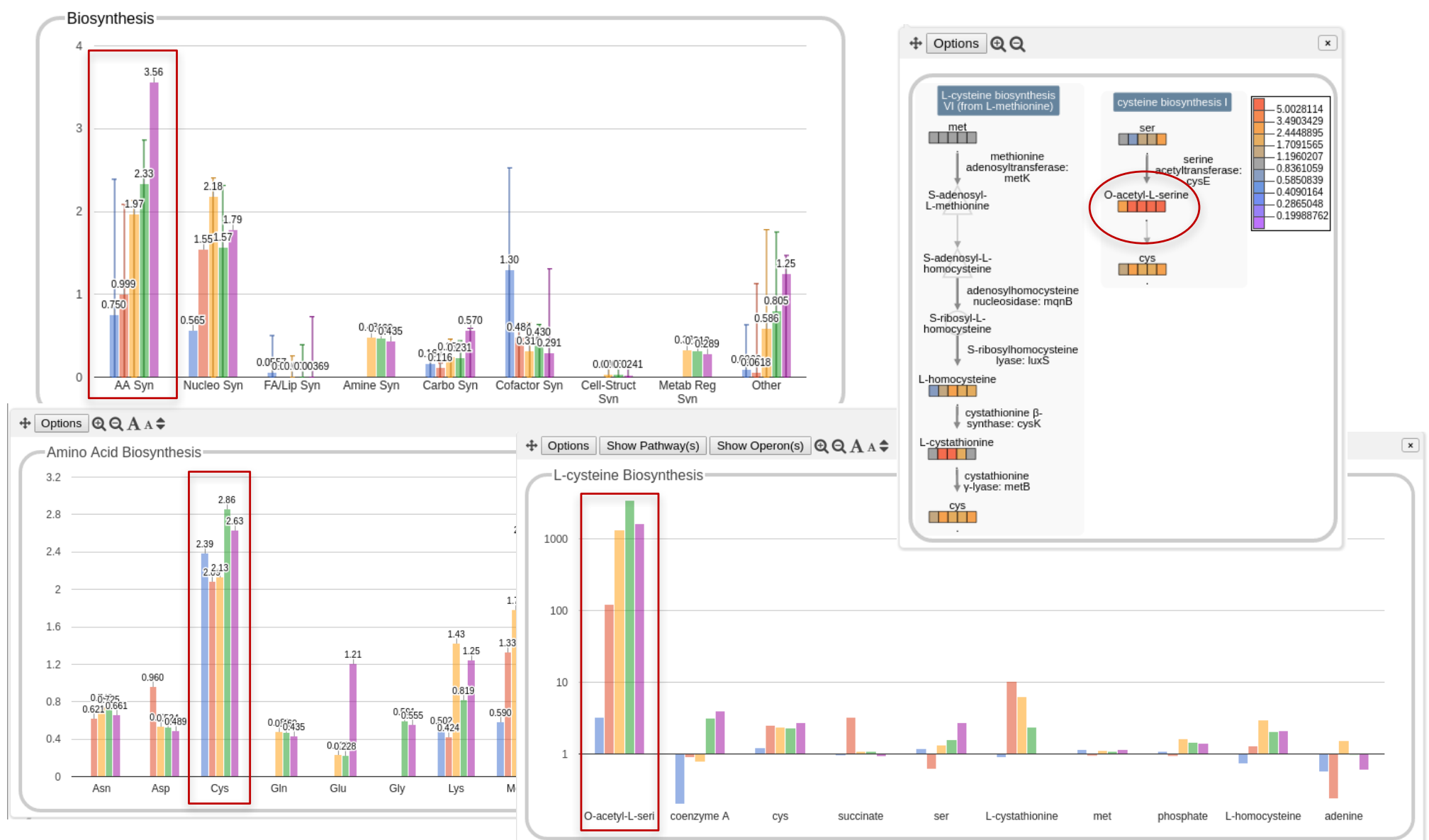




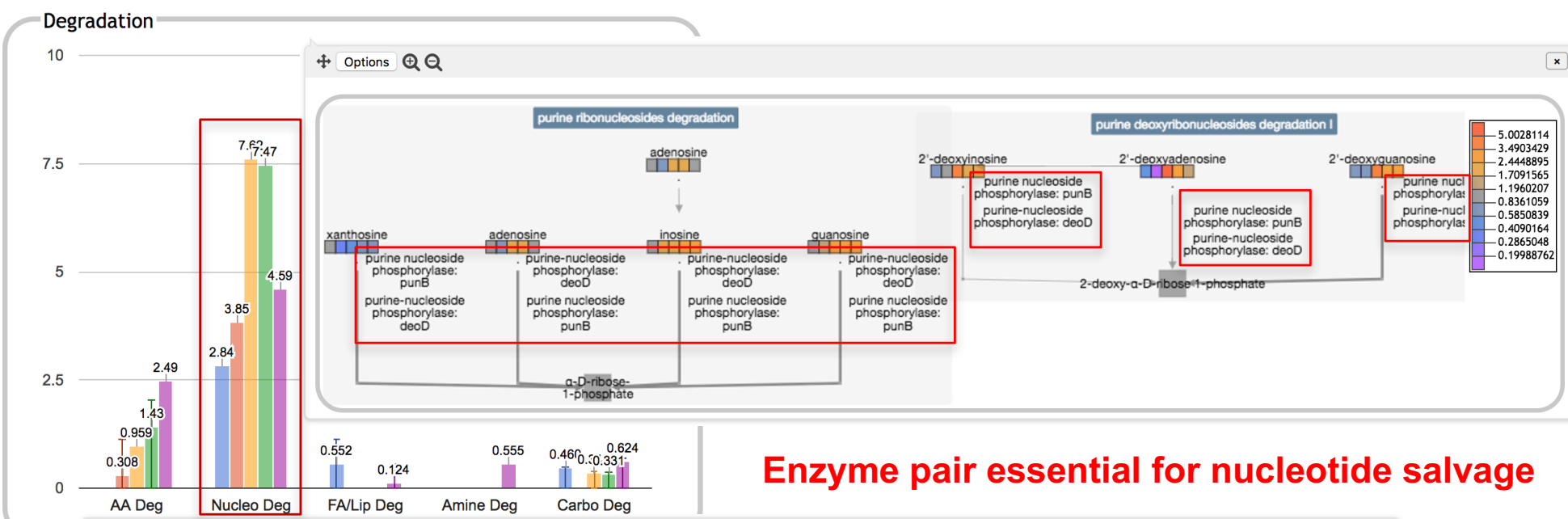

Yoptions + Options Show Pathway(s) Show Operon(s) $Q Q \mathbf{A}$ A

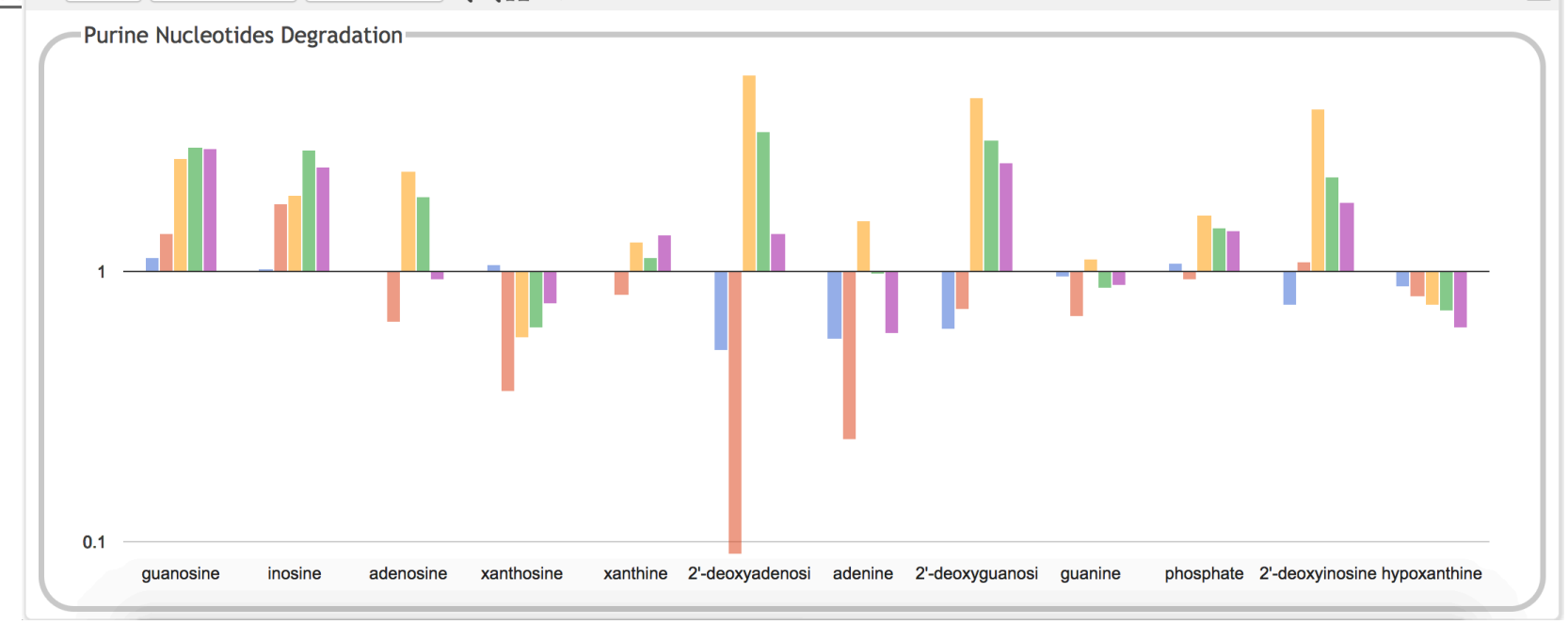




\section{Late Phase Action Reveals Bacterial Death}

- Accumulation of TCA cycle intermediates and arginine in later time points consistent with increasing morbidity

\begin{tabular}{l|l|l}
4 Options Show Pathway(s) Show Operon(s) & $Q$ & $Q$ A A
\end{tabular}
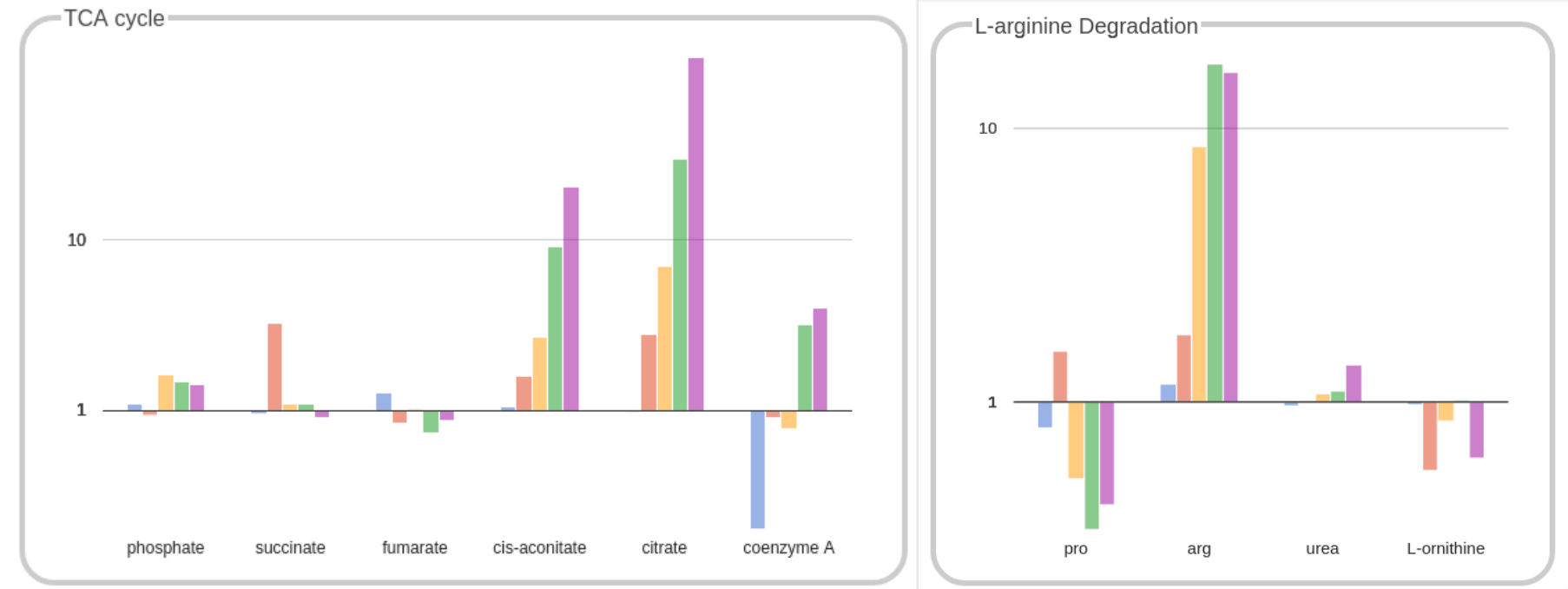


\section{New Insights from Dashboard Analysis of Metabolomics Mode of Action Study}

- Early phase metronidazole action: disruption of sulfur amino acid biosynthesis and nucleolotide degradation pathways

- H. pylori critically depends on nucleotide salvage ${ }^{1}$

- Nucleoside phosphorylase (chokepoint enzyme) evident in Dashboard

- Genome stability is tightly linked to nucleotide metabolism²

- Metabolic basis for DNA breakage

${ }^{1}$ Liechti G, Goldberg JB (2012) Helicobacter pylori relies primarily on the purine salvage pathway for purine nucleotide biosynthesis. J Bacteriol 194: 839-854

${ }^{2}$ Kunz BA, Kohalmi SE, Kunkel TA, Mathews CK, McIntosh EM, Reidy JA (1994) International Commission for Protection Against Environmental Mutagens and Carcinogens. Deoxyribonucleoside triphosphate levels: a critical factor in the maintenance of genetic stability. Mutat Res 318: 1-64 


\section{Pathway Tools Software}

- Create and maintain an organism database integrating genome, pathway, regulatory information

- Computational inference tools

- Interactive editing tools

- Query and visualization

- Generate metabolic flux models for organisms and organism communities

- Interpret omics datasets

- Comparative analysis

- Licensed by 7,000+ groups - free to academics 


\section{Pathway Tools}
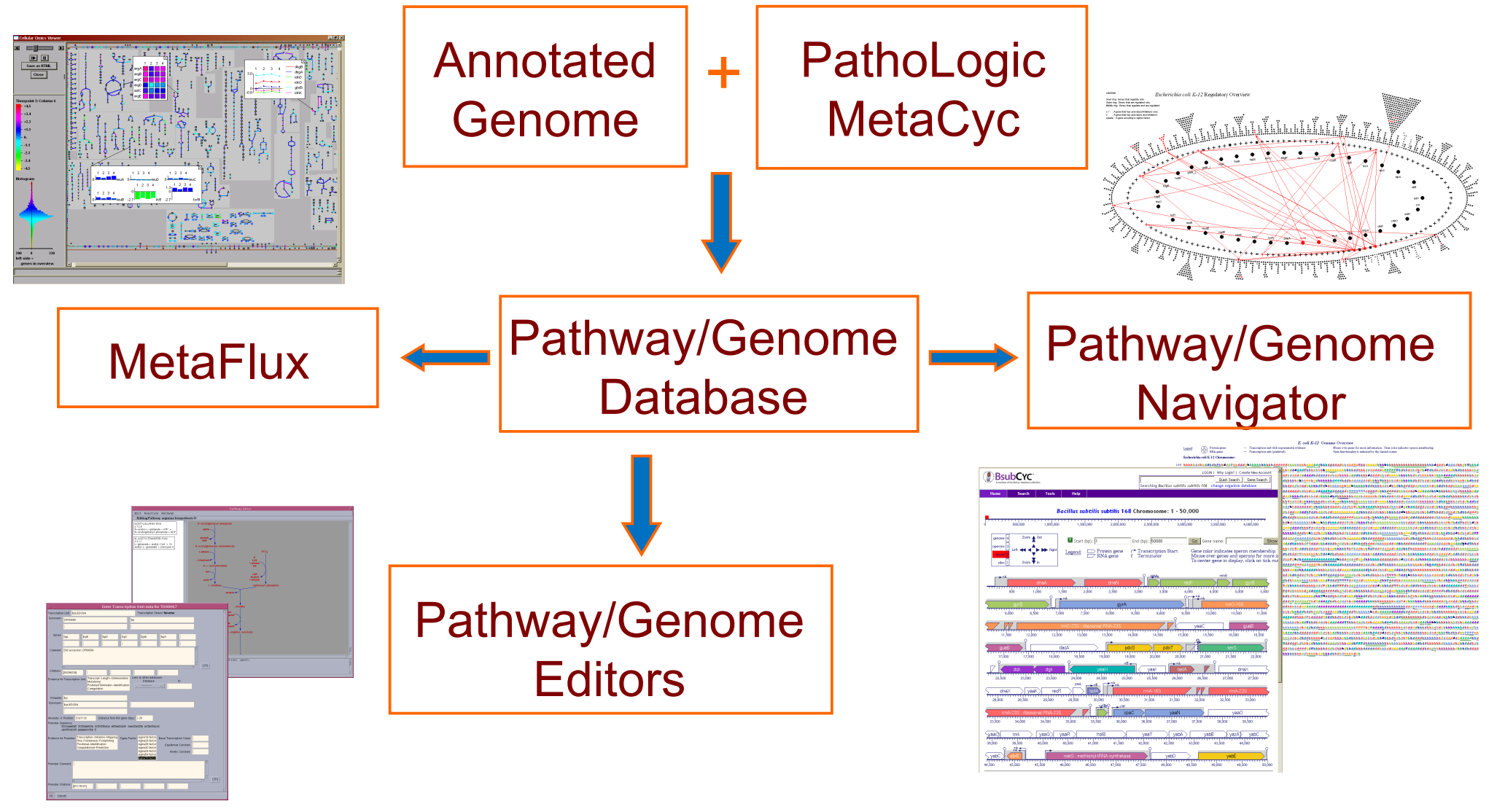

Licensed by 7,000+ Groups 


\section{Pathway/Genome Database Organization}

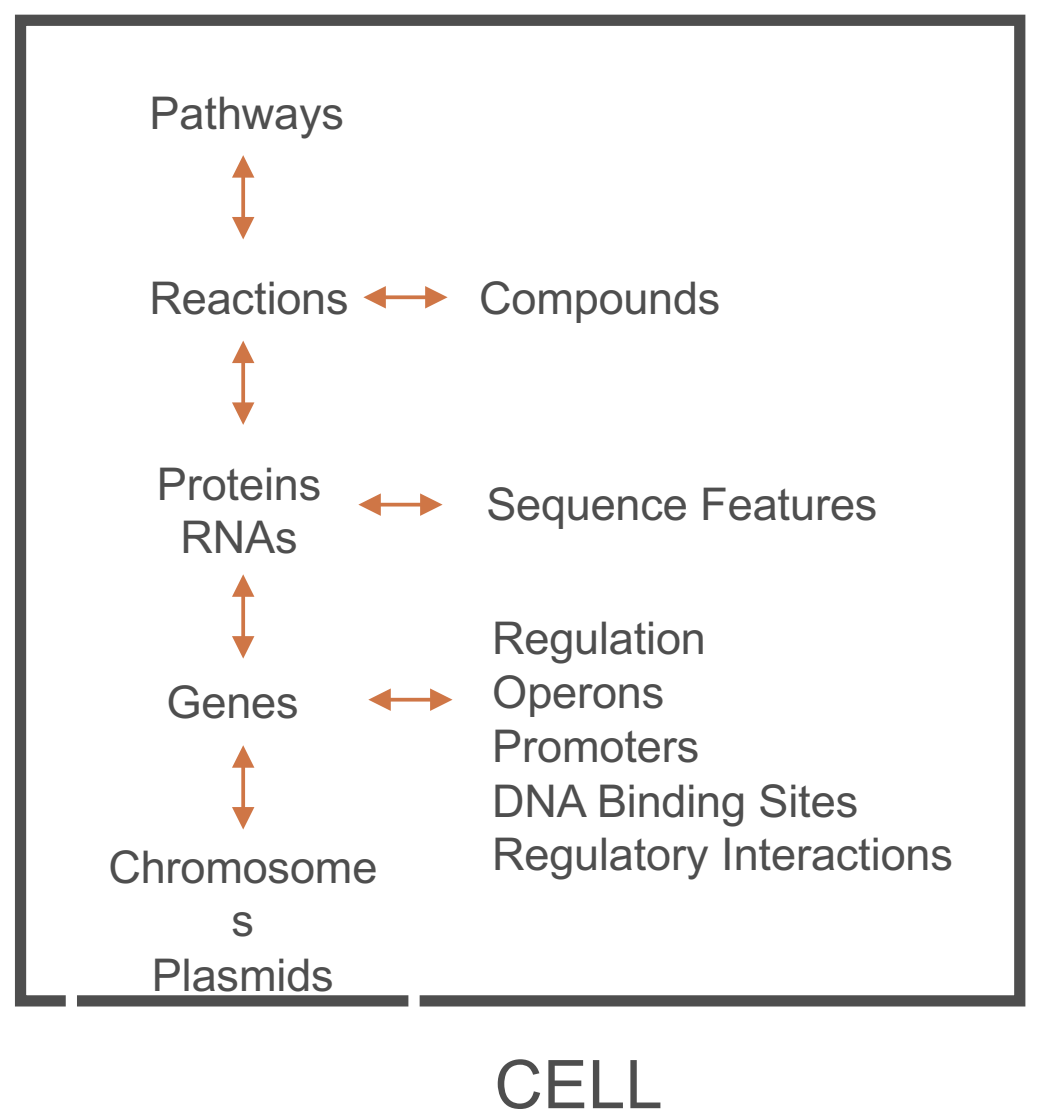




\section{BioCyc.org Overview}

- 9,300 Pathway/Genome Databases for sequenced organisms

- Predicted metabolic reactions, pathways, metabolomes

- Atom mappings, metabolic models

- Curated from 66,000 publications

- Extensive bioinformatics tools for metabolomics analysis

- Paint metabolomics data onto individual pathways, multi-pathway diagrams, full metabolic maps

- SmartTables to manipulate metabolite sets (e.g., map to pathways)

- Karp et al, Metabolites 5:291 2015
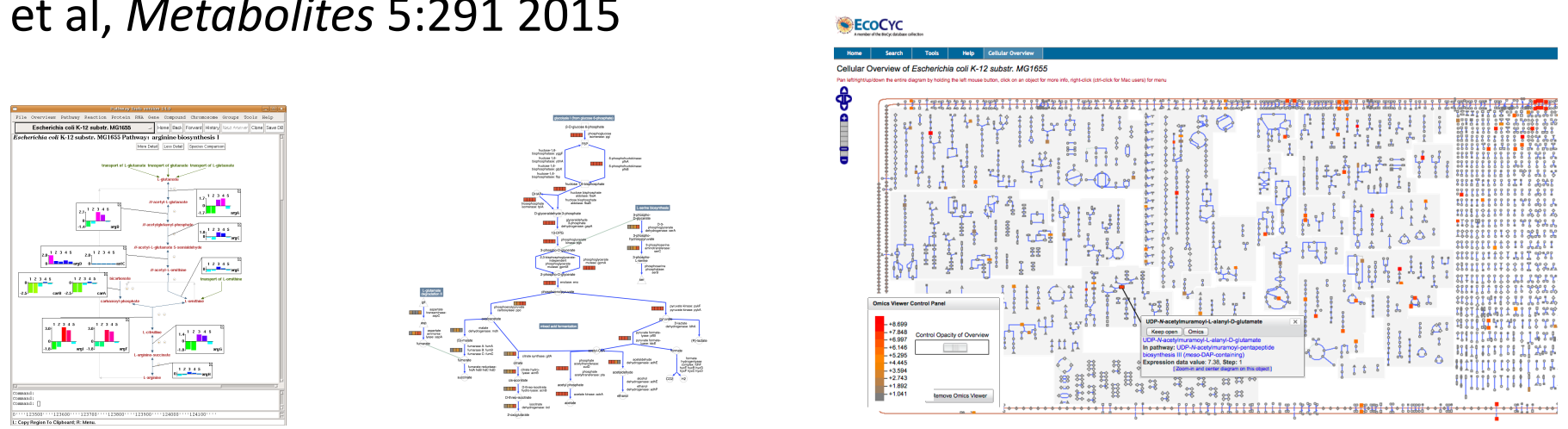


\section{Cellular Overview for Homo sapiens}

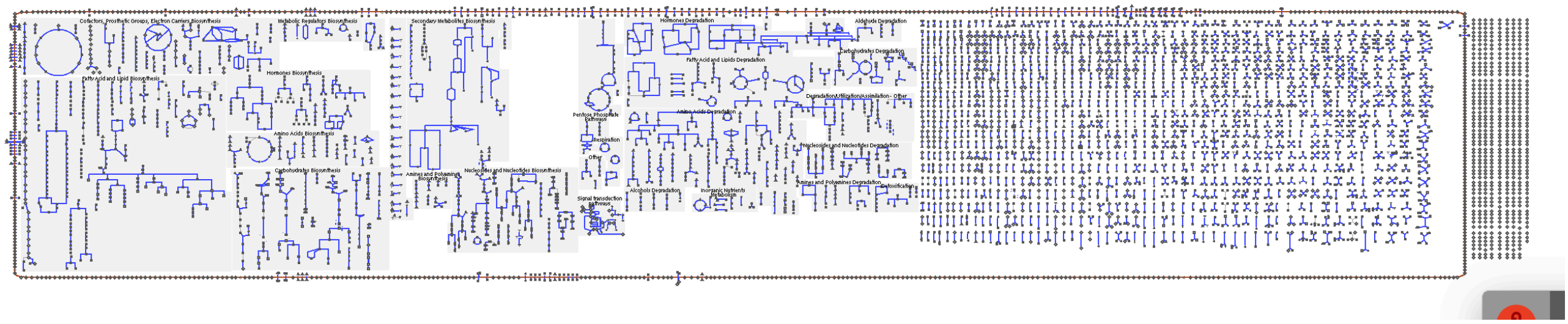


\begin{tabular}{|c|c|c|c|c|}
\hline Iome & Search & Tools & Ielp & Celluareovemew \\
\hline
\end{tabular}

\section{Cellular Overview of Homo sapiens}

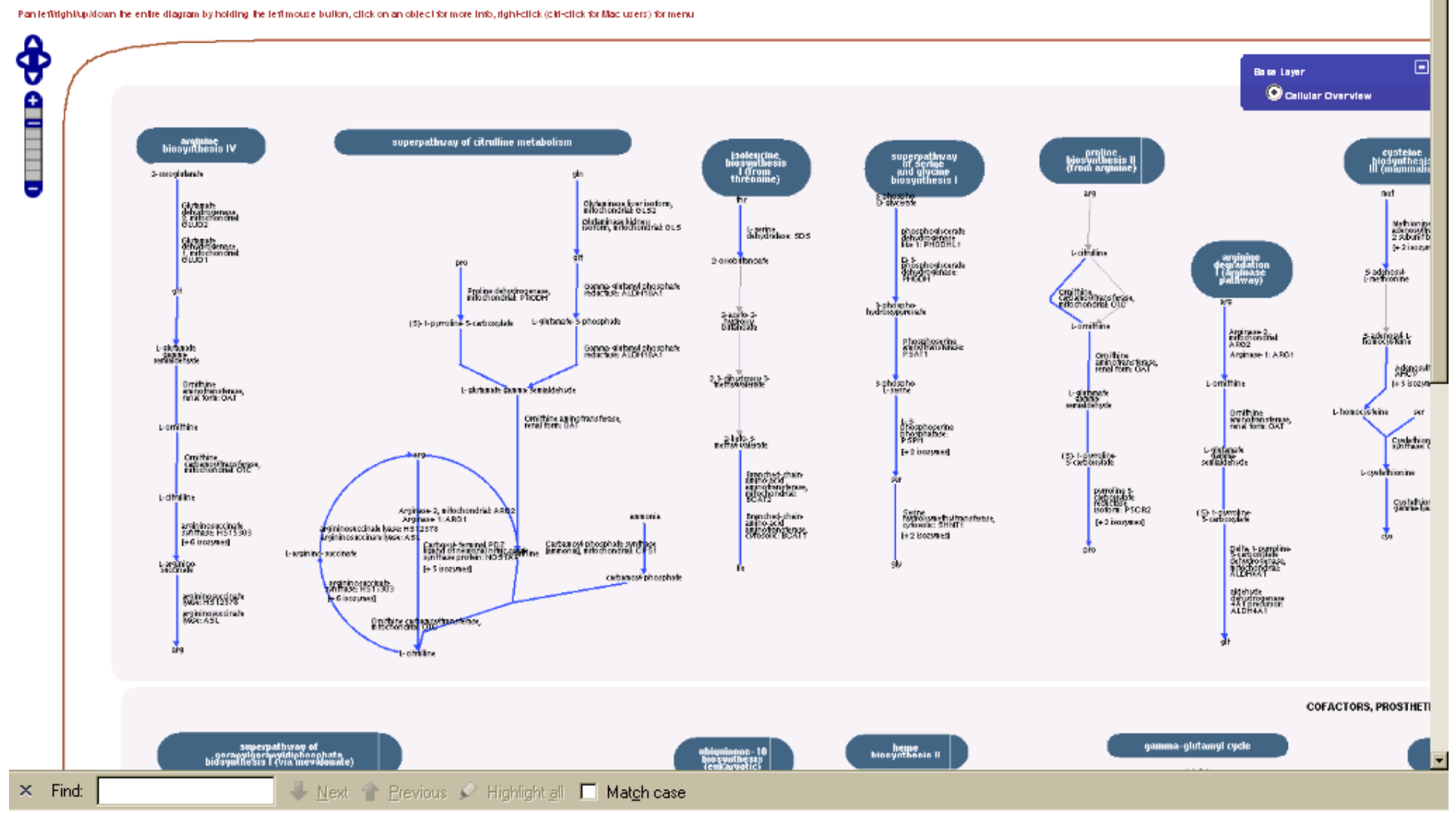




\begin{tabular}{|l|l|l|}
\cline { 2 - 3 } Cellular C & \multicolumn{3}{|l|}{ Omics Viewer Control Panel } \\
Pan leftright/ur & \multicolumn{3}{|c|}{$|c|$} \\
\hline
\end{tabular}

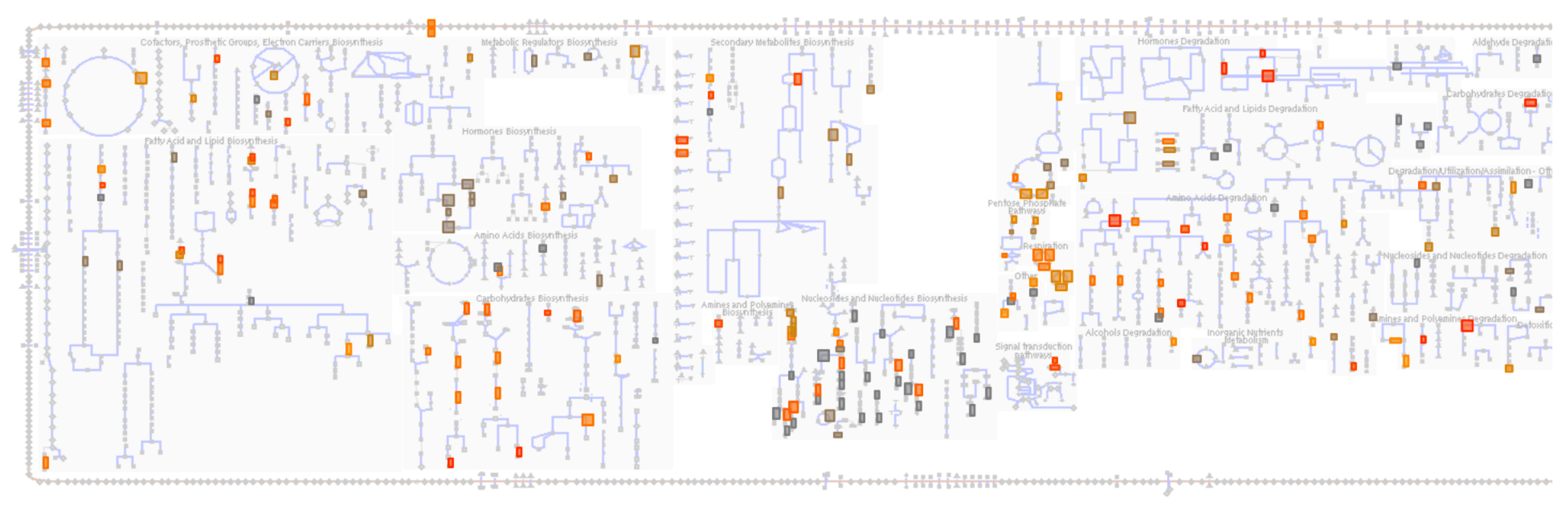




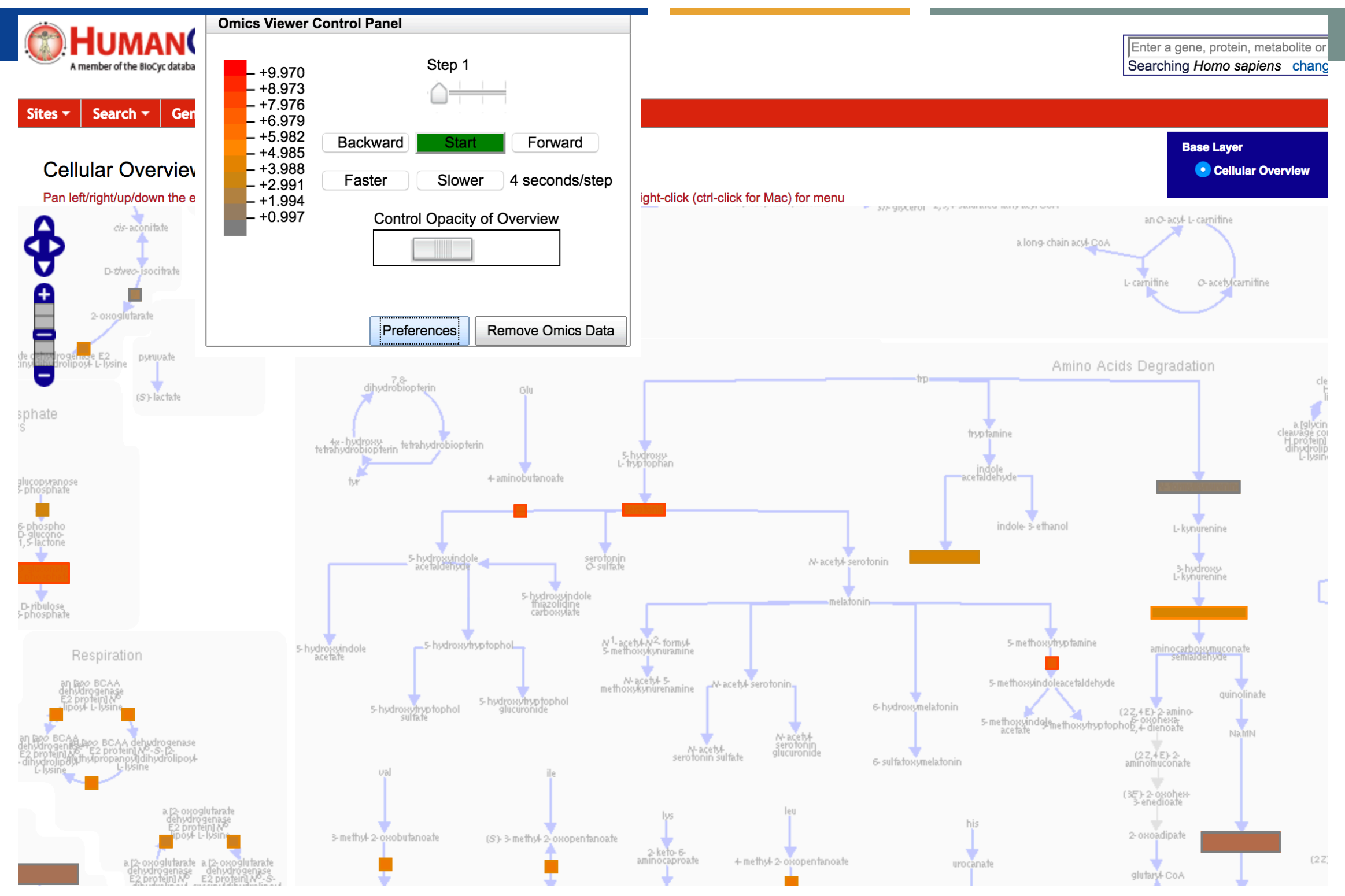




\section{Cellular Overview of Homo sapiens}

Pan left/right/up/down the entire diagram by holding the left mouse button, click on an object for more info, right-click (ctrl-click for Mac) for menu
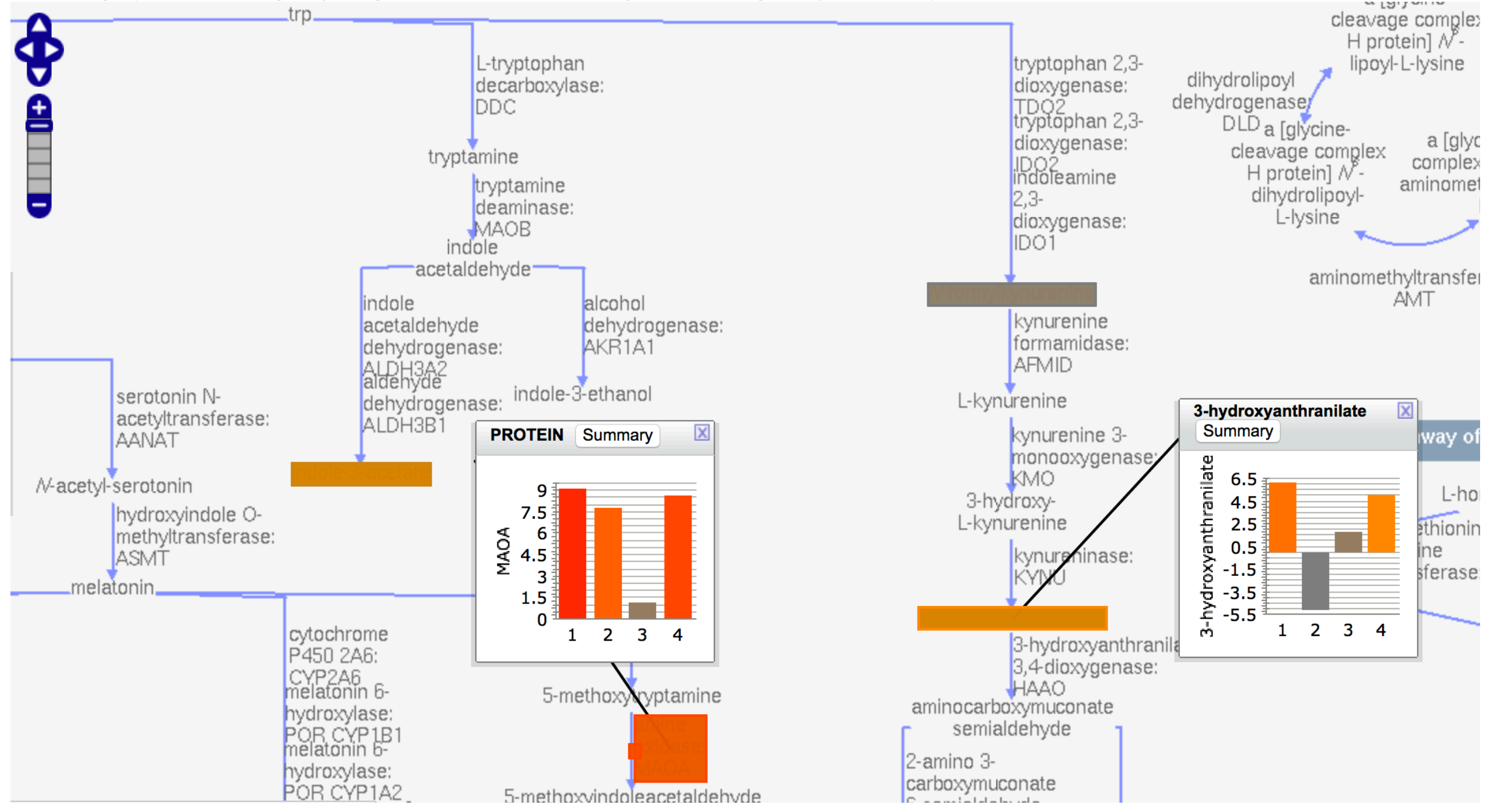

Base Layer

ptophan 2,3

dioxygenase:

Doteamine

2,3

dioxygenase:

drogenase

DLD a [glycine- $\quad$ a [glyc comple protein] $N-$ aminome pydrolipoylL-lysine

aminomethyltransfe 


\section{Summary}

- Omics Dashboard provides top-down, organism-wide, system-level view of metabolomics results

- Quickly identify and drill down to cellular systems of interest

- Uniquely well-suited to visualization of enrichment analysis results

- Complements other forms of metabolomics data analysis

- Available at BioCyc.org

- Free access to EcoCyc (E. coli) and MetaCyc

- Subscription required for access to other organisms

- Also available within Pathway Tools software

- Freely available for academic research 
Acknowledgements

- Dashboard implemented by Suzanne Paley

-H. pylori dataset and analysis courtesy of Paul O'Maille
-Funding sources:

- NIH National Institute of General Medical Sciences

http://www.ai.sri.com/pkarp/talks/

BioCyc webinars:

biocyc.org/webinar.shtml 